\title{
Can the Universe be closed?
}

\author{
Astronomers hanker after the notion that the density of the Universe is the minimum required to \\ prevent indefinite expansion, but the evidence is not all on their side.
}

ONE recurring theme in cosmology is that the density of the Universe should be exactly equal to the critical density separating universes that expand forever from those that eventually collapse back on themselves. Oddly, there has never been any astronomical evidence that this is in fact the case, and indeed there is plenty of evidence that the density is less than the critical value. But there is enough leeway in the observations to accommodate the prejudice that the value of $\Omega$, the ratio of the cosmic density to the critical value, really ought to be one.

The traditional way to find $\Omega$ is to measure the mass of as many different things as possible (galaxies, groups of galaxies, clusters of galaxies), count how many of them can be seen and then to do the appropriate multiplications. The simplest route to the mass of a galaxy is to make some assumptions about the kinds of stars it contains, when its light output is a measure of its mass. But the masses of galaxies can also be derived from their dynamical interactions, and the clear result is that most objects in the Universe are heavier than they look. Several arguments suggest that galaxies carry with them haloes of unseen dark matter.

A widely accepted, but more arguable, extension of this view is that the ratio of dark to visible matter (familiar as the mass-to-light ratio $M / L$ ) is greater for larger groups of galaxies, implying that the best estimates of $\Omega$ will come from observations of the richest clusters. The lore at present is that the mass-to-light ratio on these largest scales is one or two hundred times as great as the corresponding value for the Sun. If $\Omega$ is to be one, this ratio has to be several hundred, perhaps even a thousand, but on the other hand, it is by no means clear that $M / L$ as a function of scale has reached an asymptote even in the richest clusters of galaxies. There is still room for $\Omega=1$.

But now there may be a little less room, according to L. Cowie, M. Henrikson and R. Mushotzky (Ap. J. 317, 593; 1987). A direct way to determine the mass of a rich cluster is to measure the density and temperature profile of the hot gas that is smoothly distributed between the galaxies and which is recognizable by the X-rays it emits. The gas traces the gravitational potential, which is related to the mass distribution by a Poisson potential equation.

The difficulty is that the temperature profile of the gas cannot be deduced directly; many previous estimates of cluster mass have been based on the assumption that the gas is isothermal, which does not fit the $\mathrm{X}$-ray observations. Cowie and his colleagues do not claim to have an exact model of the gas distribution, but by taking various models, and checking that they can fit the optical data, they find that the mass-to-light ratios of clusters need be only a little greater than 100 and may be as small as 60 . It is no surprise that better modelling should lead to altered values, and the change is not huge. Its significance is that the density needed to close the Universe may be five or ten times the density of dark matter even in the richest clusters.

Even if absolute determinations of the density of clusters were possible, they would not unambiguously reveal the overall density of the Universe, which is why cosmologists have been looking for ways of finding $\Omega$ directly. The increasing refinement with which the Universe is mapped has allowed some intriguing if not yet convincing estimates. Thus, if gas can trace the gravitational potential in a cluster, why should not galaxies trace the gravitational potential in the Universe?

Improved astronomical techniques have delivered the redshifts as well as the positions of large samples of galaxies. Redshift translates into distance according to Hubble's law, so that the catalogues of galaxies listed by their two-dimensional position on the sky have been turned into catalogues of galaxies in three-dimensional space. So why not divide the mass by the volume to obtain large-scale $\Omega$ ?

There is a circular argument to unravel at the outset. If the galaxies were distributed regularly, the Universe would expand regularly and redshifts could be converted precisely into distances. But in reality, galaxies are clustered, and their velocities are affected by the dynamics of clustering on top of the cosmological expansion, so that their redshifts are contaminated by their peculiar motions, which are in turn of crucial importance in tracking the large-scale dynamics of the clusters... This seems an impossible tangle: to get the positions of galaxies, the peculiar velocities must be subtracted; but to find the peculiar velocities, the spatial distribution of the galaxies must be known.

Luckily, a little ingenuity allows some unravelling. In a dense cluster, galaxies have a characteristic velocity distribution that is independent of position; each redshift contains a random component so that, when redshift is taken as a proxy for distance, the cluster appears to be elongated along the line of sight. But, for widely separated galaxies, it has usually been assumed that the peculiar velocities are of no significance because the Hubble velocities are so much bigger. N. Kaiser has now shown (Mon. Not. Roy. Astr. Soc. 227, 1; 1987) this plausible reasoning to be false, at least in one important case.

Kaiser defines the acceleration vector as an integral over a specified volume weighted according to the density fluctuations of the galaxy distribution. Then, at a point within the volume, the peculiar velocity induced by gravitational nonuniformity is the product of the acceleration vector and a known function of $\Omega$. So, by measuring the galaxy distribution in our vicinity and knowing (as we do) our velocity relative to the microwave background, $\Omega$ should be calculable.

Sadly, Kaiser shows that this procedure does not work. Estimating the local acceleration vector requires that the positions of all neighbouring galaxies should be known, and it has been customary to take redshift as a measure of distance. But when Kaiser corrects the integral for the acceleration vector for the peculiar motion of local galaxies, the correction is disconcertingly comparable in size with the vector itself - unless $\Omega$ is much less than one.

This result is disappointing but not surprising. Both the acceleration vector and the peculiar velocities arise from departures of the large-scale galaxy distribution from uniformity. Kaiser lessens the disappointment by describing a more reliable, if more difficult, way of finding $\Omega$ on a large scale.

The proposal is that the statistics of galaxy distribution should be measured separately along and across the line of sight. Provided that the underlying distribution has no preferred direction, the difference between the two should represent the contamination by peculiar motion. A sufficiently precise analysis should yield a value of $\Omega$ directly related to large-scale cosmological dynamics. As if to sustain the spirits of the " $\Omega$ must be one" school, Kaiser also says that a simple modelling of peculiar velocities in the (local) Virgo cluster is not inconsistent with a Universe in which the critical density is exactly one. It is equally not inconsistent with $\Omega=0 \cdot 2$. David Lindley 